\title{
543.
}

\section{ON AN IDENTITY IN SPHERICAL TRIGONOMETRY.}

[From the Messenger of Mathematics, vol. I. (1872), p. 145.]

IN a spherical triangle, writing for shortness $\alpha, \beta, \gamma$ for the cosines and $\alpha^{\prime}, \beta^{\prime}, \gamma^{\prime}$ for the sines, of the sides: also

we have

$$
\Delta^{2}=1-\alpha^{2}-\beta^{2}-\gamma^{2}+2 \alpha \beta \gamma
$$

$$
\cos A=\frac{\alpha-\beta \gamma}{\beta^{\prime} \gamma^{\prime}}, \quad \sin A=\frac{\Delta}{\beta^{\prime} \gamma^{\prime}},
$$

with the like expressions in regard to the other two angles $B, C$ respectively.

Hence

$$
\cos (A+B+C)=\cos A \cos B \cos C-\cos A \sin B \sin C-\& c .
$$

$$
=\frac{(\alpha-\beta \gamma)(\beta-\gamma \alpha)(\gamma-\alpha \beta)-\Delta^{2}(\alpha+\beta+\gamma-\beta \gamma-\gamma \alpha-\alpha \beta)}{\left(1-\alpha^{2}\right)\left(1-\beta^{2}\right)\left(1-\gamma^{2}\right)} .
$$

The numerator is identically

$$
=(1-\alpha)(1-\beta)(1-\gamma)\left[\Delta^{2}-(1+\alpha)(1+\beta)(1+\gamma)\right],
$$

viz. comparing the two expressions, we have

$$
\begin{aligned}
(1-\alpha)(1-\beta) & (1-\gamma) \Delta^{2}-\left(1-\alpha^{2}\right)\left(1-\beta^{2}\right)\left(1-\gamma^{2}\right) \\
& =(\alpha-\beta \gamma)(\beta-\gamma \alpha)(\gamma-\alpha \beta)+\Delta^{2}(-\alpha-\beta-\gamma+\beta \gamma+\gamma \alpha+\alpha \beta)
\end{aligned}
$$

or, what is the same thing,

$$
(1-\alpha \beta \gamma) \Delta^{2}=\left(1-\alpha^{2}\right)\left(1-\beta^{2}\right)\left(1-\gamma^{2}\right)+(\alpha-\beta \gamma)(\beta-\gamma \alpha)(\gamma-\alpha \beta),
$$

which is the identity in question and can be immediately verified. We have thus

and thence

$$
\cos (A+B+C)=\frac{\Delta^{2}-(1+\alpha)(1+\beta)(1+\gamma)}{(1+\alpha)(1+\beta)(1+\gamma)},
$$

$$
\begin{aligned}
& 1+\cos (A+B+C)=\frac{\Delta^{2}}{(1+\alpha)(1+\beta)(1+\gamma)}, \\
& 1-\cos (A+B+C)=\frac{2(1+\alpha)(1+\beta)(1+\gamma)-\Delta^{2}}{(1+\alpha)(1+\beta)(1+\gamma)}
\end{aligned}
$$

giving at once the values of $\cos ^{2} \frac{1}{2}(A+B+C), \sin ^{2} \frac{1}{2}(A+B+C), \sin (A+B+C)$, and $\tan ^{2} \frac{1}{2}(A+B+C)$ : these are known expressions in regard to the spherical excess. 